$11 \mid 2018$

Écrire le fleuve Congo après Conrad

\title{
Tlemcen ou la source des sources
}

Hervé Sanson

URL : https://journals.openedition.org/coma/2909

DOI : 10.4000/coma.2909

ISSN : 2275-1742

\section{Éditeur}

Institut des textes \& manuscrits modernes (ITEM)

\section{Référence électronique}

Hervé Sanson, "Tlemcen ou la source des sources », Continents manuscrits [En ligne], 11 | 2018, mis en ligne le 15 octobre 2018, consulté le 14 janvier 2023. URL : http://journals.openedition.org/coma/ 2909 ; DOI : https://doi.org/10.4000/coma.2909

Ce document a été généré automatiquement le 14 janvier 2023.

\section{(c) (i) $(9)$}

Creative Commons - Attribution - Pas d'Utilisation Commerciale - Pas de Modification 4.0 International - CC BY-NC-ND 4.0

https://creativecommons.org/licenses/by-nc-nd/4.0/ 


\title{
Tlemcen ou la source des sources
}

\author{
Hervé Sanson
}

1 À l'origine, quelle origine? Pourquoi un livre s'intitulant Tlemcen ou les lieux de l'écriture? Et tout d'abord, quel titre stupéfiant ! Quelle équivalence s'établit donc ici ? Il s'agirait d'un lieu se nommant Tlemcen - au singulier. La conjonction de coordination ou instaure alors un double effet, une double possibilité : elle peut en effet revêtir un tour disjonctif - et les deux termes s'exclure l'un l'autre -, ou a contrario revendiquer une équivalence entre les deux termes - équivalence alors signifiée entre un nom propre au singulier et un pluriel commun anonyme. Disjoindre : c'est encore manière d'aiguiller les passages, de garder le caractère incomparable de chaque élément tout en les rapprochant, de maintenir une tension dans la tenue ensemble des contraires. En les liant - déliant. Tlemcen s'avère d'emblée ambivalent et toujours plus d'un; la source et l'étymologie en attestent : «Tlemcen est un pays de sources, son nom même l'annonce d'emblée de jeu : issu du berbère tilmas, au pluriel tilmisân, il veut dire sources. ${ }^{1}{ }^{~}$ Le nom même de Tlemcen confond singulier et pluriel, recèle en son sein - à première vue singulier - la marque du pluriel. Mais le titre recèle plus d'un tour en ses sources : la deuxième copule qui n'est autre qu'un double génitif-de-désigne un double engendrement de l'écriture. Les lieux de l'écriture apparaissent en effet comme les lieux où l'on écrit, où l'écriture s'est exercée et d'autre part, comme les lieux dont on écrit et que l'écriture évoque. L'ouvrage propose alors dans sa thématique un mouvement centrifuge: le lieu où l'on écrit apparaît dès les premières pages de Tlemcen. «LE CADRE PREMIER DE MES ÉCRITURES fut cette cour, ce que nous Algériens appelons le centre de la maison [...] $]^{2}$.» 
figure 1 : Le patio par Philippe Bordas.

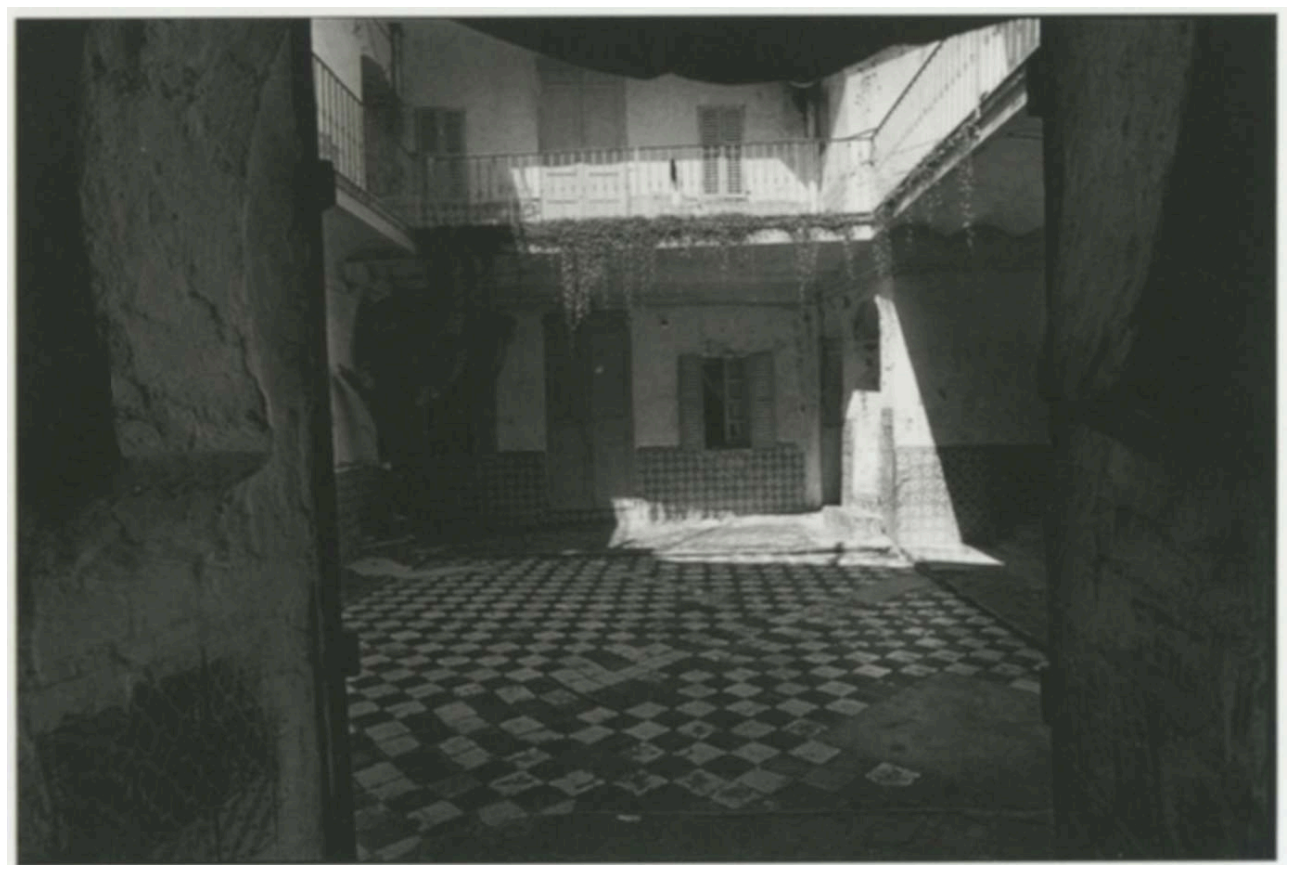

Avec l'aimable autorisation de Philippe Bordas et de la Revue Noire, éditeur de l'ouvrage Tlemcen ou les lieux de l'écriture.

2 L'écriture a pour lieu tout d'abord le centre de la maison, "cette cour ", mais un phénomène singulier advient alors : l'écriture se scinde, se divise, se dissémine en plus d'une («MES ÉCRITURES»), et cache, en son sein, la pluralité de sa pratique et sa nature bifide, entre artisanat et art. La notion d'artisan, chère à Dib, permet de convoquer l'ouvrier, celui qui œuvre de ses mains, celui qui possède un métier, justifiant ainsi l'emploi par Dib de l'expression «mes exercices d'écriture», qui pointe ici le fait d'acquérir progressivement la pratique d'une discipline en se soumettant à certaines règles. Un mouvement centrifuge se dessine - disions-nous - autour de cette cour de maison car les autres lieux évoqués de Tlemcen sont les lieux précisément dont on écrit, et ils se déploient géographiquement autour de la maison familiale du jeune Dib.

\section{Entre temps : un ouvrage qui revient de loin}

3 L'ouvrage fait la navette entre les photographies de Mohammed Dib datant de 1946 et les photographies de 1993, signées Philippe Bordas. L'écriture assure le lien entre les époques; là se situe précisément le lieu de cet ouvrage : entre temps. Un entre temps aura passé : au premier dépôt le temps aura sédimenté d'autres couches, entre temps aura littéralement fait œuvre: les temps se font alors face, se confrontent. À la lettre, l'ouvrage se déroule entre temps : il a lieu $(x)$ entre le cahier de photographies de 1946 en ouverture et le cahier de 1993 en clôture. Il a lieu(x) entre les temps et pose de surcroît en sa composition, en sa progression formelle, la question du passage de la pratique amateuriste à la pratique professionnelle, de la pratique personnelle - pour son propre compte - à la pratique artistique - celle qui fait œeuvre. En effet, le jeune Dib en 1946 est un photographe amateur qui entame tout juste une carrière littéraire en publiant en revue alors que Philippe Bordas en 1993 est un jeune photographe "au talent confirmé », nous indique la quatrième de couverture, un photographe professionnel ${ }^{3}$. 
L'ouvrage a donc lieu(x) entre les temps et revendique un parti pris éthique en même temps qu'esthétique : le photographe Dib - infans (muet, immature) -, qui n'avait pu capter les «Trois-Frères » en temps de brouillard en 1946, laisse place au photographe confirmé de 1993 pour appréhender une réalité algérienne spécifique. En 1946, quelque chose peinait à se dire, quelque chose échappait et trouvait un rapprochement inédit avec le contexte socio-politique de l'époque : les événements du 8 mai 1945 sont encore dans toutes les mémoires et ont profondément marqué les indigènes. Certains historiens, tels Annie Rey-Goldzeiguer, s'accordent désormais à reconnaître cette date comme le commencement de la guerre d'indépendance nationale. En 1946, en apparence, la situation est revenue au calme, mais clandestinement, les nationalistes, notamment ceux de l'ex-PPA (Parti du Peuple Algérien) dirigé par Messali Hadj, s'apprêtent à fonder l'O.S. (Organisation secrète), prélude à la constitution du FLN (Front de libération nationale) et préparent déjà le déclenchement de l'insurrection nationale. La photographie manquée de Dib en 1946 fait écho à une réalité souterraine, latente, pour l'heure difficilement préhensible. Ainsi, le terme "mes écritures" employé par Dib corrobore l'idée de latence, d'essai, de préparation. Pour le jeune écolier, il s'agissait d'abord d'explorer les capacités de la langue de l'autre, de la découvrir au fur et à mesure.

Visiter d'abord l'instrument, l'éprouver, en explorer le registre: je n'aspirais à guère plus, mais cela m'avait pris dès les bancs de l'école. Déjà je me lançais dans des exercices d'écriture 4 .

Un parcours s'amorce alors : nous y reviendrons et notamment sur la composition de l'ouvrage. L'écrivain algérien vivant en France, dans tous les cas, délègue son regard à un jeune photographe français et ce faisant, (re)noue avec une histoire familiale: l'éditeur Jean-Loup Pivin accueille dans son catalogue un écrivain ayant côtoyé son père, José Pivin, dans l'Algérie coloniale des années cinquante autour de la revue Soleil, où le jeune Dib publia sa première nouvelle, «Un après-midi d'été ». Une manière de croiser dans la variation les parcours, et de faire de l'écriture un fil conducteur entre deux époques, deux lieux : de l'Algérie coloniale des années cinquante jusqu'à la France des années quatre-vingt-dix. Ici, précisément, le syntagme "les lieux de l'écriture » révèle son ambivalence. L'écriture qui avait lieu dans le patio de la maison tlemcenienne s'est donc excentrée, conférant alors au titre une nouvelle profondeur : Tlemcen porte en son sein - déjà - la France. L'ambivalence est ici conservée car la France peut trouver place dans une équivalence excluante, disjonctive, Tlemcen ou bien les lieux de l'écriture, c'est-à-dire la France, mais aussi être comprise dans un rapport d'équivalence absolue, de correspondance. Reste : l'écriture, et la permanence de son agent : la personne physique de l'écrivain algérien d'expression française Mohammed Dib autour duquel ont gravité d'abord José Pivin puis aujourd'hui son fils Jean-Loup Pivin. Ce qui se crée ici est le déport d'un regard, une prise de contact à distance, une procuration au sens où Philippe Bordas apparaît tel le membre agissant de Dib, comme si celui-ci lui avait conféré légalement le pouvoir d'agir en son nom (selon le Robert historique : 2953). Le noir et blanc joue de la confusion entre les photographies de Dib et celles de Bordas car quatre clichés de ce dernier trouvent place au fil de l'ouvrage, échappant au cahier constitué en fin de volume. C'est donc bien à un regard de contrebande qu'il nous semble avoir affaire, un regard qui passe en contrebande, jouant un certain travestissement, "changeant de vêtements afin de n'être pas reconnu " (comme le Robert historique l'indique à l'entrée «travesti »: 3903). L'articulation entre texte et photographie met au jour une problématique du reste et de la trace. 
DU TLEMCEN DONT TEMOIGNENT CES PHOTOGRAPHIES prises en 1946, peut-on dire qu'il ne reste que le nom ? Ce serait sans doute aller trop loin. Il y a pourtant quelque chose de cela ${ }^{5}$.

5 L'écriture s'enjoint alors de convoquer la ruine, d'évoquer ces lieux disparus : ce quelque chose qui reste. Quelque chose : l'écriture tourne autour du nom. Le nom fait lien, assure la continuité. Les majuscules exhaussent ainsi une mémoire légendaire (legenda: ce qui doit être lu), légende des photographies accompagnant le texte, mais avant tout cette ruine de l'écriture - performée - dont il ne reste que le nom, mémoire en dépôt sur la page cryptée en un signifiant emblématique, ruine érectile, érigée en son corps propre ; reste le dit du lieu-dit. Reste : ce qui se dit. Ce qui s'écrit. Précisément, l'écriture tente de se tenir à l'angle de ce qui a déjà disparu et de ce qui reste : nous dressant ainsi à la lettre son portrait en jeune ruine, donné à lire, voir, entendre. Une mémoire en souffrance joue l'inter/ruption, la rupture entre un présent de la photographie à l'œuvre du témoignage -tel l'effet de réel de la photographie qu'évoque Barthes dans $L a$ Chambre claire - et le temps-passé-de-leur-prise, soulignant ainsi la prise en compte du passage du temps et la ruine-à-l'œuvre. Le passage des majuscules aux minuscules déploie le champ différentiel des temps, les performe en quelque sorte, et permet ainsi leur mise en regard. C'est un ouvrage qui revient de loin, amorçant sa constitution dès 1946, un ouvrage qui procède par couches successives, qui fait œuvre après-coup mais qui est d'abord le fruit d'un certain (dés)œuvrement. Il renouvelle ainsi une certaine visée de la photographie, illustrant ce que Derrida nomme une fantômaticité ou bien encore une hantologie : " tout commence avant de commencer. ${ }^{6}$ " À l'origine, Dib réfute l'origine : reportons-nous à la première séquence de Tlemcen intitulée « «Les lieux de l'écriture » » et à ses premières phrases :

AU COMMENCEMENT EST LE PAYSAGE, - s'entend comme cadre où

l'être vient à la vie, puis à la conscience.

À la fin aussi.

Et de même, dans l'entre-deux ${ }^{7}$.

Cette série d'assertions à caractère itératif annule précisément le point d'origine puisque le paysage ne possède pas de référence temporelle précise et que, de plus, il précède l'individu - origine de la conscience - et se fait point de départ de l'œuvre d'art. C'est qu'en effet, l'ouvrage revient de plus loin que lui-même, dès avant sa conception. La photographie se hante elle-même, par son dispositif d'ouverture/clôture dans le recueil, se re-double, performe le mouvement de l'écriture en ruine - mais en jeune ruine - car quelque chose a valeur proleptique dans l'acte même d'écrire. En constatant le caractère ruiniforme de ce dont elle témoigne (perte et résistance de ce qui reste tout à la fois), l'écriture dans le même tour le réaffirme, l'érige en un monument pour la mémoire - une matérialité sans matière, dirait Derrida ${ }^{8}$. Dib dénonce dans L'Arbre à dires cette fantômaticité de l'image photographique, cette capacité de la photographie à receler des fantômes.

Quand bien même j'y figurerais, une photographie atteste la réalité sans moi. Mais quelle réalité est-ce que cette réalité ectoplasmique, cette réalité qui vous procure l'impression bouffonne et inquiétante d'être peuplée de fantômes? Vidant gens et choses de toute vie, les gelant pour l'éternité, qu'est-ce ?'

7 Tlemcen ou les lieux de l'écriture performe donc ce que Dib théorise dans «L'homme en proie aux images »: la photographie ne garde pas la vie, contrairement à l'opinion commune, mais au contraire, elle vous dépossède de toute trace de vie et vous pétrifie dans une immanence réductrice. 


\section{Rendre les lieux à eux-mêmes : de la trace photographique}

8 En écho à la réflexion dibienne, Philippe Bordas désertifie l'image photographique ; le cahier de 1993 comporte très peu de photographies avec des individus pris de face. Des ombres, des dos tournés, un musicien absorbé par son instrument et ne nous renvoyant pas notre regard, une femme voilée - fantôme blanc pris à la marge de la photographie, à la dérobée - constituent ce cahier. Les deux seuls individus nous renvoyant notre regard sont une vieille femme, vestige de 1946, d'une époque révolue, et un jeune garçon de ferme, appartenant lui aussi à une Algérie rurale, traditionnelle. En fait, le regard nous est refusé. L'observateur-lecteur de l'image photographique est confronté à la vacuité d'un monde comme si le photographe cherchait à traduire la perte irrémédiable d'un espace-temps déterminé. Ce faisant, le lecteur-voyeur est renvoyé à sa propre vacuité et est conduit à s'interroger sur la notion de ruine autour de laquelle évolue le travail photographique de Philippe Bordas. L'impression de déjà-vurenforcée par le choix du noir et blanc - conditionne notre lecture du cahier de 1993 et met donc en place une hantologie de l'image photographique, hantologie de l'Être liée à une poétique de la différance travaillant l'ensemble de l'ouvrage. C'est que le photographe a changé d'identité entre temps : le photographe algérien de 1946 a laissé place au photographe français de 1993. Peut-être s'exprime-t-il, pour ce dernier, le refus de reconduire un regard ethnologisant, hérité de la période coloniale. Malek Alloula avait analysé dans son ouvrage Le Harem colonial, devenu une référence obligée, la mise en scène de la photographie coloniale, support de la carte postale coloniale. $\mathrm{Ce}$ regard ethnologisant s'accompagnait de la projection sur l'Autre d'un fantasme de nature sexuelle. Philippe Bordas, conscient de l'héritage qu'il véhiculerait à son insu et de la filiation dans laquelle il pourrait malencontreusement s'inscrire, évite soigneusement toute mise en scène, toute prise de vue trop étudiée: le mouvement imprimé à ses clichés et le caractère "sur le vif » de ses photographies s'écartent résolument de l'héritage colonial. Avec la photographie page 145 du cahier final, Bordas s'inscrit en faux contre l'esthétique de la photographie coloniale : les deux voiles sont saisis en marge, quasiment hors champ, proposés à l'œil du spectateur dans l'incomplétude que permet la mobilité des deux figures. 
Figure 2 : Les voiles blancs

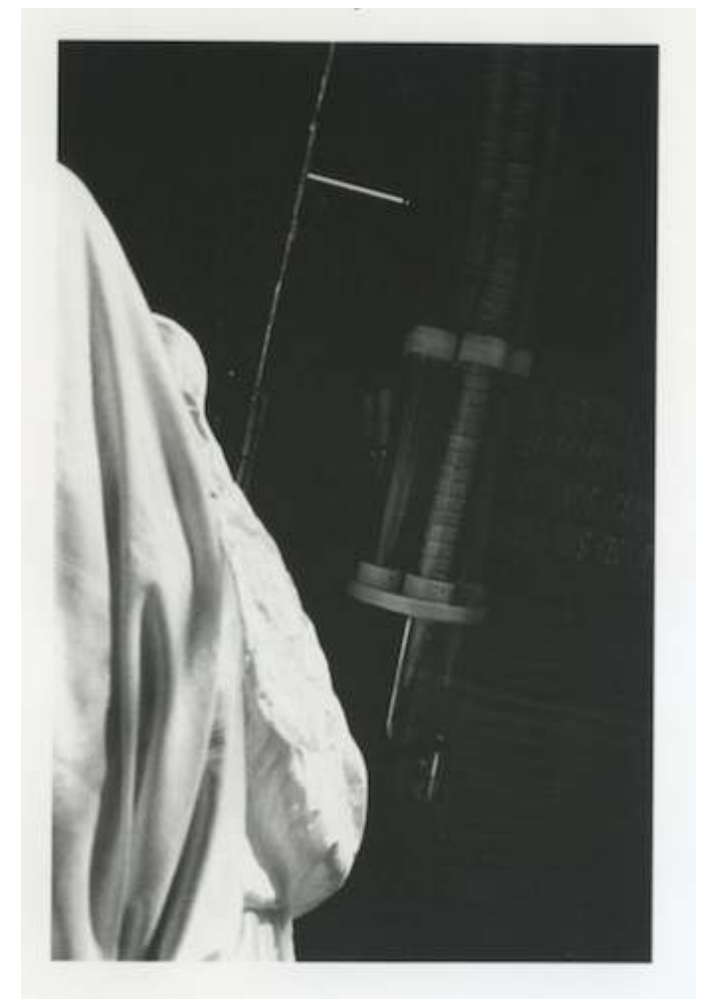

Avec l'aimable autorisation de Philippe Bordas et de la Revue Noire, éditeur de l'ouvrage Tlemcen ou les lieux de l'écriture.

Le photographe réussit à nous transmettre une réserve face à la «capture " photographique de l'autre, et la pudeur que sa pratique photographique manifeste. Ce qui nous est donné à voir c'est: ce qui échappe, la trace de ce qui passe. Walter Benjamin, dans son étude "Petite histoire de la photographie », analyse les premières photographies comme le témoignage d'une classe sociale spécifique. L'aura de ces clichés, leur hic et nunc, "trame singulière d'espace et de temps", émane d'une conjonction entre la technique et son objet.

Ces images sont nées en des lieux où chaque client voyait dans le photographe d'abord un technicien de la nouvelle école, tandis que le photographe voyait en chaque client un représentant de la classe montante, pourvu d'une aura qui se nichait jusque dans les plis de sa redingote ou de sa lavallière ${ }^{10}$.

Dib nous fait la radiographie d'une société agonique, bientôt bouleversée par des mutations socio-économico-politiques irréversibles. Un photographe comme Atget introduit, selon Benjamin, une conception et un usage novateurs de la photographie, ouvrant la voie aux "découvertes» de la photographie surréaliste. Ainsi la photographie s'entend-elle désormais à capter la déliquescence des lieux, et la perte d'aura qui leur est attachée. Mais aussi à rendre les lieux à eux-mêmes. À leur propre présence. La photographie saisit alors les fantômes et tente de les matérialiser pour l'observateur. Les photographies du jeune Dib se situeraient entre les clichés d'un Hill au dix-neuvième siècle, en ce qu'il présente une Algérie traditionnelle, coloniale et bientôt caduque - en fait un portrait hic et nunc-, et les clichés d'un Atget au tournant du vingtième siècle. Philippe Bordas, par la procuration dont il bénéficie, entend prolonger la démarche dibienne, la mener à son terme en quelque sorte. En désertifiant l'image photographique, il rend les lieux à eux-mêmes, et applique les leçons d'un Atget 
en soumettant à notre sagacité les lieux d'un crime. Au regard de cette hantologie de la photographie, emblématique apparait la section intitulée " Les Trois-Frères ». D'entrée, le paradoxe de la photographie est posé : la précision de 1993 permet de capturer par temps de brouillard les fantômes des « Trois-Frères ", leur aspect brouillé, effacé.

figure 3 : Les Trois-Frères

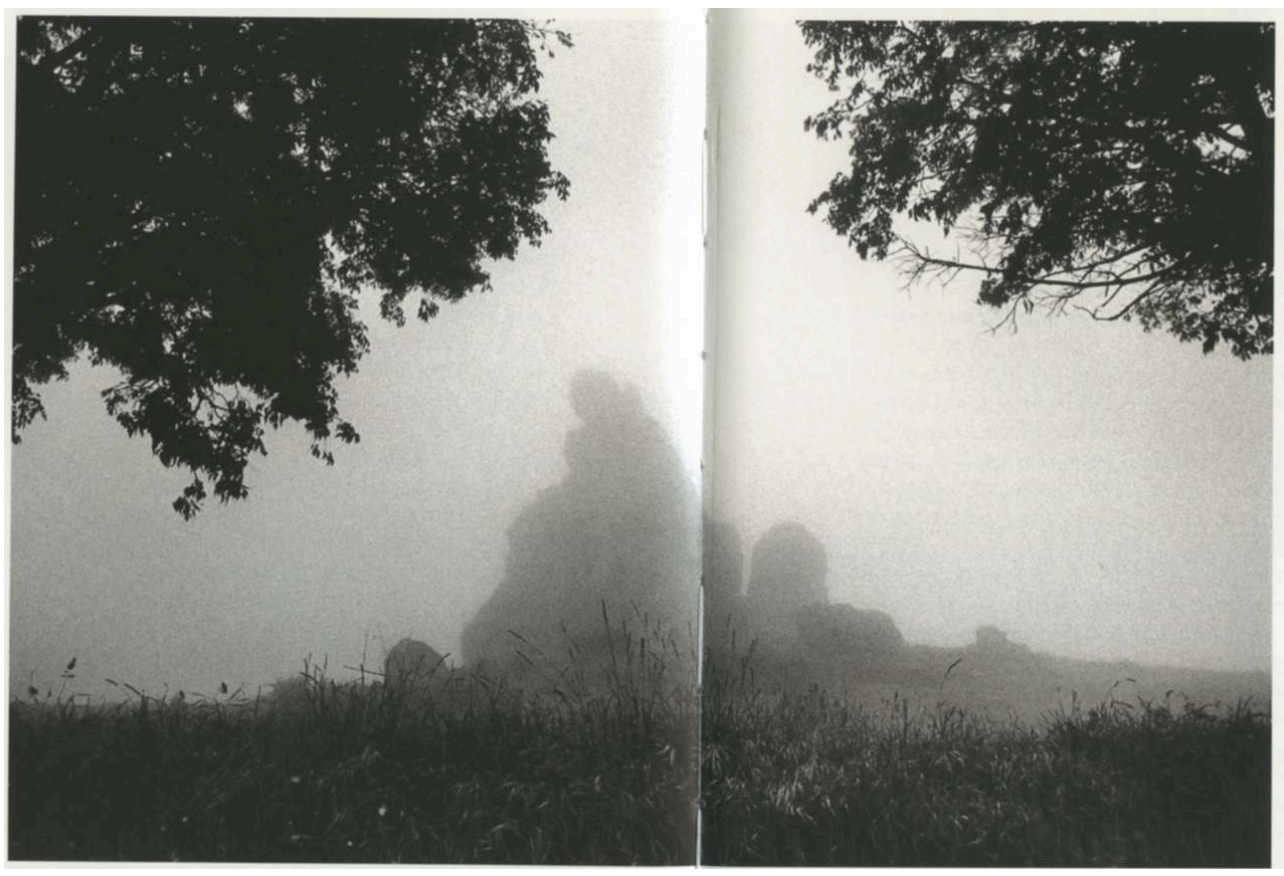

Avec l'aimable autorisation de Philippe Bordas et de la Revue Noire, éditeur de l'ouvrage Tlemcen ou les lieux de l'écriture.

Dib en pointe précisément «l'atmosphère paradoxale : car pris par un temps qui ne participe pas aux clichés habituels ${ }^{11} »$. La technologie permet donc ici de restituer la fantômaticité des lieux, de ces témoins particuliers. Et plus encore : elle engendre ses fantômes, révèle l'ambivalence constitutive de l'écriture et de ses lieux. Un extrait de L'Incendie travaille d'ailleurs le jeu de contraste entre le blanc et le noir : «Ce piton, blanc d'un côté, noir de l'autre, gardait la route..$^{12}$ » Les "Trois-Frères » ne sont pas à proprement parler préhensibles, identifiables, ils échappent à toute classification, mêlant influences mythiques et influences sacrales: ils convoquent les pouvoirs du muthos, de la fable, en tant qu'ils sont objets de fabula, de "on-dit», mais peuvent revendiquer une filiation divine en tant qu'ils sont "voués, comme malédiction, à une divinité » et ainsi consacrés en poésie (selon le Robert historique, à l'entrée « sacrer » : 3348). L'écrivain joue ici la polysémie de certains termes, garante du caractère ambivalent de l'écriture. Il ne cesse de favoriser la faculté de tisser des liens, de tracer des analogies, de permettre à la langue de faire feu de tout bois, de travailler l'équivocité des syntagmes. En outre, il s'ingénie à pratiquer l'art de l'oxymore dans cette séquence, instaurant un art du contretemps, de la temporalité dé/jouée.

En été, sous un soleil de fin du monde souvent, ils offrent l'apparence de mythiques et derniers voyageurs traversant un désert, mais de voyageurs arrêtés sur leur chemin, de voyageurs cloués par la malédiction qui frappe ceux que l'heure a devancés ${ }^{13}$. 
Une apocalypse qui est aussi une révélation. Les lieux sont toujours soumis à d'autres temps, le voyage s'effectue toujours immobile à grands pas, pour reprendre la belle expression de Paul Valéry dans "Le Cimetière marin ", reprise par Claude Simon (je fais référence à son ouvrage Orion aveugle paru en 1970). Ici s'inscrit le déni de toute perspective téléologique ; un écartèlement pèse sur ledit passage : la phrase se retrouve soumise à des tensions contraires.

\section{« Immobile à grands pas »: l'écriture en ses chutes}

13 La phrase dibienne se fait "carrefour de sens ", expression que Claude Simon utilise pour qualifier la progression d'Orion aveugle qui «avance à tâtons dans la forêt des signes ${ }^{14} »$. Ainsi, la phrase piétine, acquérant un équilibre par la conjonction des forces contraires qui la composent. Elle se règle sur la structure même de l'ouvrage. Mohammed Dib nous joue ici un de ces tours dont il a le secret : la "zone centrale " qu'il questionne restera inentamée, nouée mais préservera l'enfance de l'art en même temps que l'art de l'enfance en évitant d'en présenter une image figée, statufiée, définitive. Le portrait d'une mémoire en ses errances advient au plus fidèle. Dans la quête incessante d'elle-même et le pari du recommencement.

Les lieux sont soumis à une tension énergétique multipliant les croisées temporelles, les déplacements, les reprises de la phrase sans cesse sous correction. De leur nomination dénuée de sources rationnelles et avérées, les « Trois-Frères » subissent la malédiction, c'est-à-dire la mauvaise diction. Et Dib de préciser : « À y regarder de près, ils restent pourtant distincts, tout soudés qu'ils semblent ${ }^{15}$. "Soudés en apparence, ils se distinguent les uns des autres. Ces mal nommés se révèlent jouets du malentendu - mal entendus.

En place de la route de Sebdou, à peu de distance au-dessus de Mansourah, ils s'élèvent et demeurent, témoins non pas sourds peut-être, mais muets d'une vie qui survit dans le seul cisaillement des cigales ${ }^{16}$.

Quoi qu'il en soit de la tension temporelle que symbolisent les "Trois-Frères", de la logique disjonctive/rapprochante, l'accent y est porté d'une faculté à entendre car le témoin est d'abord celui qui entend et s'y entend à survivre. Littéralement, les « TroisFrères » demeurent, jouant le temps contre le Temps monumental, institutionnel. Art du contretemps, tout comme le volume en son entier joue la stratégie du contretemps. Comme le cliché le montre, les «Trois-Frères » dé/jouent le temps du cliché habituel, exhaussent un temps paradoxal, ils sont pris par Bordas mal à propos. Ils s'affirment synecdochiques d'une facture, d'un art de faire caractérisant l'ensemble de l'ouvrage. Le constat se révèle alors sans appel: "Le Médresse, ou si l'on préfère, le beylick, détruit, c'est un des lieux de l'écriture détruit ${ }^{17}$.» L'écriture doit alors combler l'intolérable faille ${ }^{18}$, croître sur la ruine. Épouser la ruine : l'écriture dibienne emploie le terme chance ( "L'espoir et le désespoir d'y arriver sont la chance de l'écrivain ${ }^{19}$ ») en ses multiples variations. Ce qui se trouve ici souligné a pour nom : l'écriture en ses chutes. L'écriture doit s'y entendre afin de bien tomber. Face à l'intolérable faille à combler, l'écrivain n'a d'autre recours qu'en l'art de la chute. Par ailleurs, Dib convoque la notion de juste mesure, de bonne distance pour une juste saisie du monde.

L'ÉCRITURE EST UNE FORME DE SAISIE DU MONDE. Mais cette saisie s'effectue dans un mouvement de recul, - recul du scripteur par rapport au monde et recul du 
même par rapport à l'écriture. L'œuvre, semble-t-il, se constitue dans ce creux, dans cette distance ${ }^{20}$.

Cette saisie du monde doit compter avec la facture de la phrase, sa composition : une «phrase bien faite » joue le compromis du savoir-faire poïétique et de l'événement incalculable. Quoi qu'il en soit, l'écriture pour Dib est toujours sujette à méprise potentielle. À mal entendu. L'ambivalence de l'écriture est convoquée à divers titres : objet de curiosité, mauvaise interprétation, à la lisière du licite, du convenable.

L'une d'elle me suggéra, un jour :

- Tu devrais nous composer des amulettes que nous porterions contre le mauvais

œil, et nous te donnerions alors du sidi ${ }^{21}$.

7 L'œil dissémine le texte : l'écriture peut-elle trouver le bon œil, celui qui serait à la fois source et œil-témoin? L'écriture est invoquée ici comme une protection contre le mauvais œil ; bonne sorcellerie, elle revendique donc son équivocité, elle donne bonne garde à celui qui en bénéficie, pointant son 'ayn, c'est-à-dire sa double acception source/ œil. Monnayable contre du sidi. C'est-à-dire, là encore, contre de l'ambivalence. Le sidi en effet est certes un vin célèbre d'Algérie, et à titre coranique prohibé, haram, mais aussi un titre religieux conféré à certains individus.

Ce mot saint, j'y pense, risque d'induire en erreur. Il n'est que la traduction impropre d'un terme, wali, dont le sens serait plutôt serviteur, ou d'un autre terme, morabith, qui veut dire habitant d'un ribat (lieu clos, couvent). Mais ce wali ou morabith prend dans la conscience religieuse rang de maitre, avec l'acception de guide spirituel $^{22}$.

Et précisément le sidi développe la trame de l'erreur (étymologiquement : " errer çà et là ») en liaison avec l'écriture et la traduction. L'ambivalence du sidi proposé au jeune Dib par ces femmes s'en trouve doublée : le sidi n'est ainsi pas celui qu'on croit - de prime abord. Partant, Dib pointe la vanité de toute traduction, et qu'il lui en échappera toujours quelque chose. Une herméneutique de l'ex-propriation, de l'écart se fait jour : l'écriture prend des tours et détours pour proposer une lecture (trans)frontalière, jouant sur plusieurs niveaux de signification. Car toute traduction est par essence impropre et se veut une traversée. Un autre aspect de l'ambivalence de l'écriture réside dans sa présence/absence. En effet, Dib écrit dans un premier temps: «LE CADRE PREMIER DE MES ÉCRITURES fut cette cour, ce que nous Algériens appelons le centre de la maison $[. . .]^{23}$ ". Mais par la suite le caractère transfrontalier de l'écriture, visible/ invisible, entre présence et absence, son caractère spectral est relevé par la description dibienne :

Je m'établissais dans un angle pour me livrer à mes exercices d'écriture [...]. S'il n'y avait le groupe, qu'on voit dans la photographie ci-contre, c'est moi qu'on aurait vu. [...] Invisible, je suis pourtant là présent par la procuration du regard qui fixe la scène $[\ldots]^{24}$. 
Figure 4 : « Le patio » de Dib

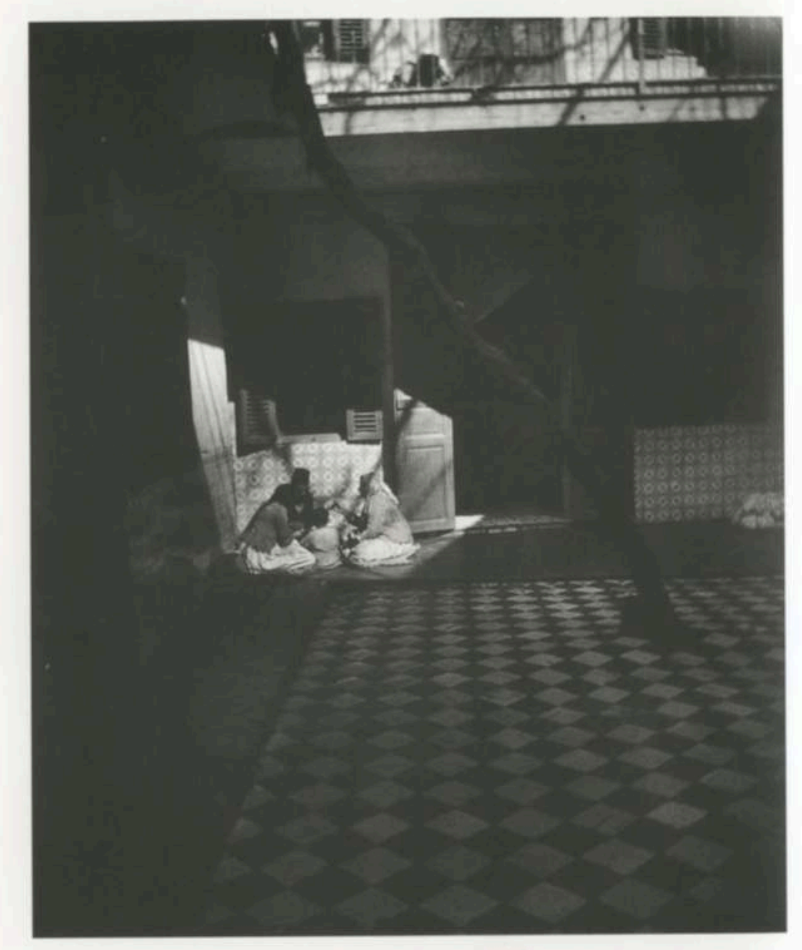

Avec l'aimable autorisation de la famille Dib et de la Revue Noire, éditeur de l'ouvrage Tlemcen ou les lieux de l'écriture.

19 Ainsi, l'écriture s'avoue hors champ: elle se pose toujours aux marges, en marge, apparaît double ; mais précisément, ce que la photographie avoue, c'est une présence en creux de l'écriture, un non-lieu de l'écriture car, ici, la photographie ne voit pas. La procuration symbolisée par le regard du photographe-écrivain qui «fixe la scène » fixe un écrire en marge, un écrire qui échappe précisément à la localisation, au lieu, aux tentatives de spatialisation qui sont autant de volontés d'identification, de classification de l'écriture. L'emploi du conditionnel et l'association oxymorique des adjectifs présent et invisible témoignent de la seule localisation viable : un « dedans de la parole qui, seul, demeure notre patrie féconde ${ }^{25} »$. Mais il y a davantage : l'écriture, par la grâce d'un incompossible (invisible / présent), est subterfuge. Le sujet même de l'écriture est hors champ, comme en atteste la description par Dib d'une photographie d'enfance, privée, qui ne nous est pas donnée à voir, hors-livre, donc inexistante pour le lecteur-spectateur. Photographie absolue, telle celle fondatrice, déclenchant l'écriture pour Marguerite Duras dans L'Amant, «scène primitive » de l'écrivain, cette photographie idéale fonde la quête ultérieure - cristallise le moment propice, le Kairos. Son absoluité ne peut que se conjuguer avec l'absence, et elle ne peut que demeurer soustraite au regard du spectateur puisqu'elle est la cristallisation phantasmatique de celui qui pratique l'anamnèse, processus par nature infini. Elle associe aussi toutes les marques d'une identité idéale-synthétique-de l'individu. Elle revendique un caractère "achevé, terminé » (selon le Robert historique, entrée "absolu»: 10) que réfute précisément le processus d'anamnèse et s'absentifie du même coup. En effet, elle ne peut qu'être invisible puisque je ne me reconnais jamais en photographie une fois pour toutes. Dib nous trace alors un autoportrait en autre, insistant sur l'aspect 
fondamentalement autre du petit garçon qui lui fait face. "C'est le phénomène qui me fait face, avec lequel je suis confronté. ${ }^{26}$ » Le sujet se dissocie ; déjà l'écriture fuit audelà. La syntaxe joue la division, la mobilité. Déjà, le sujet futur de l'écriture gagne son écart, s'isolant des autres. Afin d'insister sur le sentiment radical d'étrangeté qu'il ressent face à cet autre de soi-même, il déterritorialise le signifiant. C'est-à-dire qu'il substitue aux référents de sa société d'autres référents, exotiques, hétérogènes à la société algérienne de l'entre-deux-guerres. Les vêtements même subissent un déplacement, une mutation d'identité, ils y perdent leur idem, le véritable référent qu'ils désignent. Par exemple, « un kalpack au poil noir, à la Atatürk - le colback que portèrent les troupes napoléoniennes - lui sert de couvre-chef ${ }^{27}$ ».

Figure 5 : Mohammed Dib, enfant

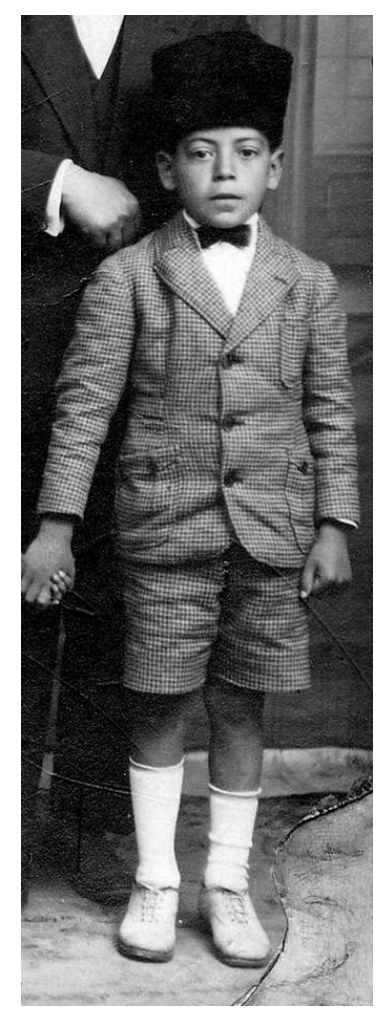

Avec l'aimable autorisation de la famille Dib

Avec ces trois identités: kalpack, colback, couvre-chef, dans une déterritorialisation continue, l'accessoire vestimentaire passe les continents et les époques, mariant les idiomes, accouplant des référents historico-culturels hétérogènes, convoquant sur la scène textuelle tel personnage historique étranger à l'Histoire algérienne (Atatürk), mettant en scène l'expulsion du sujet disant je, et annonçant la dissociation - structure spéculaire, symétrie négative - de la phrase suivante. Comment s'identifier à l'autre qui me fait face ? Le retour du pronom je marque la rupture interne du sujet qui, à la lettre, de cette confrontation, n'en revient pas. Il subit le charme, et le trébuchement. "Je n'en reviens pas, le nœud papillon surtout me réjouit. Et me scandalise ${ }^{28}$.» L'hyperbate signe ici le piège dans lequel Dib a conscience d'être empêtré. Et ce faisant, la phrase n'en revient pas: la réjouissance mène au scandale, révèle son contraire. La photographie ment : étymologiquement (selon le Robert historique : 2196) elle manque de parole; je ne s'y reconnaît jamais. Précisément, la phrase en sa progression trébuche, 
c'est-à-dire conduit au-delà d'un tronc initial et implique l'idée de déplacement. L'hyperbate «Et me scandalise » offre l'exemple saisissant de ce trébuchement. À point nommé : ça trébuche sur ce syntagme. En effet, le Robert historique de la langue française (entrée "scandale» : 3407) nous avertit que "le latin, pour traduire l'hébreu miksôl "obstacle, ce qui fait trébucher", a repris le grec skandalon "piège" (skandalê désigne le trébuchet d'un piège où se trouve placé l'appât)... » Un renversement phénoménal témoigne du mensonge inhérent à la photographie. Roland Barthes analyse dans $L a$ Chambre claire cette imposture :

Devant l'objectif, je suis à la fois : celui que je me crois, celui que je voudrais qu'on me croie, celui que le photographe me croit, et celui dont il se sert pour exhiber son art. Autrement dit, action bizarre : je ne cesse de m'imiter, et c'est pour cela que chaque fois que je me fais (que je me laisse) photographier, je suis immanquablement frôlé par une sensation d'inauthenticité, parfois d'imposture ${ }^{29}$.

Le langage même de Dib se retrouve contaminé par l'imposture photographique: le langage alors, déterritorialisé, n'en revient pas. Dib s'exprimera à son tour sur cette imposture dans L'Arbre à dires : facteur de dépossession du sujet, de division interne, la photographie ne favorise pas, selon lui, le travail de mémoire.

Pourtant, ni l'image qui me représente n'est moi, ni celle qui m'offre le spectacle de la nature n'est la nature. Pas plus moi que la nature nous ne nous y reconnaissons ; mieux, elles nous dépossèdent, moi, de moi, et la nature, d'elle-même. Sidérant les forces qui concourent à nous faire être, elles pétrifient de surcroît l'obscur travail de la mémoire comme celui de la psyché ${ }^{30}$.

Mais il y a davantage: l'écriture ne se laisse pas photographier, standardiser. L'aventure d'une écriture est toujours singulière et s'appuie sur la métaphore vive, supplément à la photographie - en témoigne la description des faïences, du cadre de la maison natale. La métaphore vive précisément s'attache à présenter «toutes choses "comme en acte" ", analyse Ricœur dans son ouvrage éponyme. "L'expression vive est ce qui dit l'existence vive $e^{31}$.» À l'encontre d'une photographie figeante, qui immobilise son sujet en objet, le classifie une bonne fois pour toutes, l'usage de la métaphore vive s'ingénie à capter le mouvement, l'énergeia de la scène, sa dynamis et la coloration de la vie. Ce que Ricœur qualifie par ailleurs de "tropes d'invention", en opposition aux tropes usuels qui correspondent aux clichés de la langue, à ses usages conventionnels.

\section{Du pouvoir de la métaphore : le témoin-fabula}

La métaphore vive aurait à cœur de déjouer les habitus de la langue morte. Il s'agirait donc d'une représentation, d'un art de la mimesis, non caricaturaux qui, à partir de la restitution d'un Réel donné, font œuvre : ils créent dans un écart qui témoigne de la possibilité pour la métaphore de saisir ce qui sans cesse se meut dans l'existence. La description offre précisément vue(s): «cela vous a l'humide transparence d'un œil d'enfant. ${ }^{32}$ " II convient de noter ce que Dib écrit dans L'Arbre à dires : "Ce qui est sûr, c'est que je suis un visuel, un œil. Cela ressort dans mes écrits et quel que soit le genre d'écrit : poème, roman, nouvelle ${ }^{33}$.» Ne perdons jamais de vue pour notre propos que Dib fut un peintre avant de devenir écrivain. L'ombre verte de la vigne, ultime, est convoquée afin de tout recouvrir de la couleur verte, dans un mouvement de verticalité "sans fin" qui donne à la description un aspect exhaussant, ascensionnel, hyperbolique, interrompu certes par la clôture de la séquence sur le plan littéral mais poursuivant sa progression dans l'esprit du lecteur. Ce que la littérature joue en 
supplément se nomme la capacité au rêve: "De quelques couleurs. Moins pour rehausser la photographie que pour donner à rêver ${ }^{34}$ "; la capacité au transport aussi : par la métaphore. La préposition à symbolise ce mouvement directionnel qui n'aboutit jamais. Il s'agirait de gagner ailleurs par la force d'évocation du signifiant. De décoller, c'est-à-dire de quitter les cadres figés, par l'écho de l'existence vive qu'inscrirait la métaphore vive. Dans la valeur mobile progressive de la préposition à se dessine le trajet d'une écriture ; c'est-à-dire une traversée (d'après l'italien tragetto) empêchant toute immobilisation, toute catégorisation - ce que la photographie induit de par sa nature. Le pouvoir de la métaphore réside dans le fait qu'elle participe à la double tension caractérisant l'imitation des actions les meilleures (la mimesis) : la soumission à la réalité et l'invention fabuleuse, la restitution en même temps que la surélévation, c'est-à-dire un déplacement vers le haut. Un enseignement nous est ainsi donné : qu'il n'y va jamais d'une politique de maitrise, de préhension, de mainmise, d'une éthique du propre. L'éthique de la littérature selon Dib se jouerait dans cette labilité, ce potentiel à l'écart, à la tenue sur le seuil, entre deux taxinomies. Ainsi du sujet de l'écriture : même quand il y est, il n'y est pas. Il prend la ligne de fuite. Il échappe en propre à la représentation figée : ainsi Dib nous peint le portrait de l'écriture en autre. Ailleurs. Sous l'écriture fonctionnelle, utilitaire, se cache dans un angle la " gratuite ", l'écriture de création.

- Tu écris des lettres?

- Non, j'écris pour moi.

De brusques rires d'incrédulité accueillaient ma réponse ${ }^{35}$.

La sorcellerie est tapie dans la syntaxe car l'emploi d'un complément d'objet indirect permet de neutraliser - sans en avoir l'air - une affirmation trop catégorique, unheimlich, pour une communauté centrée sur l'entraide et la solidarité, basée sur la fusion du je dans le nous de ladite communauté. Cette communauté pratique en quelque sorte la transitivité. La véritable réponse de Dib aurait dû être la suivante: "Tu écris des lettres?-Non, j'écris.» Le complément d'objet indirect, d'autre part, permet d'oblitérer autrui en établissant un cercle communicationnel qui aboutit en fait à une indirection majeure, affirmant l'absence de destinataire précis au principe de l'écriture, relevée par Derrida. L'écriture poursuivant son errance voit émerger son portrait : la ville n'y est que pré/texte, assise textuelle. Dib remet en question ce portrait de sa ville natale en toute fin d'ouvrage.

Ce par quoi j'aurais dû conclure - ou commencer ? - les espèces de visions, d'échos dont remue et résonne toute ma mémoire, c'eût été de dévoiler le visage de cette ville dont à chaque coin de phrase il est question [...] J'en suis toujours là, cette description restera toujours à faire. Et je m'interroge : si elle ne s'imposait pas? Dans ce cas, pourquoi la faire $?^{36}$

\section{De la description : quel témoin pour la ville?}

Dib remet en cause un certain type de description qui n'échappe pas aux catégories réalistes mais il la remet en cause en ménageant une marge d'incertitude; l'usage du conditionnel passé interroge la pertinence d'une description et bat en brèche toute conception d'une ekphrasis - ce procédé consistant à commenter une œuvre d'art en la redoublant d'un texte élevé au rang d'œuvre -, texte s'affichant explicitement comme une construction puisque les photographies prises en 1946 par l'auteur et présentées ici revendiquent leur statut amateuriste. En outre, l'ekphrasis se révèle un doublet par le 
biais d'un autre support artistique : or, la photographie y est ici pré-texte et non métatexte. En effet, la littérature joue le supplément et cette capacité au rêve, Dib l'exprime déjà dans la postface à Qui se souvient de la mer, roman édité en 1962 qui évoque d'une façon hyperbolique la guerre d'Algérie. C'est avant tout l'occasion d'une investigation de la Ville. Évoquant le célèbre tableau de Picasso, Guernica, l'écrivain pointe la fonction de l'artiste qui consiste à mettre en forme(s) un inconscient collectif : "C'est là l'aspect le plus important de cette démarche : celui d'accoucheur de rêves ${ }^{37}$. " La littérature affirme ici son potentiel maïeutique : la description de type réaliste ne permet pas le témoignage adéquat puisqu'il s'agit d'exprimer l'inconscient collectif d'un peuple, de réactiver les mythes fondateurs chez l'homme, en traduisant ses aspirations les plus profondes. Dib poursuit :

L'autre versant des choses que j'ai voulu explorer ressemble fort au mariage du paradis et de l'enfer, et il n'est possible de rendre ce qui ressemble tantôt au paradis, tantôt à l'enfer, et souvent aux deux à la fois, que par des images, des visions oniriques et apocalyptiques. Ce sont les seuls projecteurs capables de jeter quelque lumière sur de tels abîmes ${ }^{38}$.

Ainsi seule une écriture qui s'écarte du référent réaliste par l'adoption d'une facture surréaliste peut faire révélation (rappelons que le sens premier d'apocalypse est précisément révélation) mais par ailleurs, cet extrait met en lumière les pouvoirs de l'image, au sens où l'entendait Pierre Reverdy :

L'image est une création pure de l'esprit. Elle ne peut naître d'une comparaison mais du rapprochement de deux réalités plus ou moins éloignées. Plus les rapports des deux réalités sont lointains et justes, plus l'image sera ${ }^{39}$.

« Une certaine manière d'être de la ville ${ }^{40}$ » : voilà ce que vise l'écrivain. Le visage de la ville évoqué par Dib fait écho à l'étymon vis de l'ancien français signifiant «champ visuel ». Mais précisément Dib joue aux coins de la langue; plaçant son objet au petit coin, il nous dérobe le champ visuel d'un espace nommé Tlemcen. Il nous dérobe la description et nous fait comprendre que l'écriture n'advient jamais dans le champ mais dans l'angle de la prise de vues, au nom même d'un parti pris: instituer Tlemcen comme le lieu de passages, à l'image même du livre-gigogne qui est ainsi constitué. La ville est lieu de la lettre : pré-texte, elle suscite le portrait de l'écriture sous couvert de topos. Assia Djebar évoquait un pays-langue: nous habitons d'abord la langue avant d'habiter les lieux, un " "dedans de la parole" qui, seul, demeure notre patrie féconde ${ }^{41}$ ». Ce que Djebar interroge ainsi concerne l'appartenance, la nationalité de l'écrivain maghrébin, un sentiment irrémédiable d'exil à l'égard de sa communauté :

Par ailleurs, la notion même de «nationalité littéraire » serait à ré-éclairer : est-ce que, durant vingt siècles, l'écrivain du nord de l'Afrique ne serait-il pas, de facto [...] un métèque dans la cité ? Un étranger, plus encore dans l'étrangéité de sa condition que, par exemple, pour n'avoir pas pleuré à l'enterrement de sa mère ? ${ }^{42}$

L'écrivain maghrébin, promis à l'expatriation depuis quelques décennies, se voit donc le mieux placé pour remettre en cause cette notion de "nationalité littéraire ». Il est amené à se faire passeur dans la langue et témoigne d'une appartenance exclusive, celle de la langue qui nous fonde et nous définit, alors que «l'écrivain du Sud ne sera jamais plus porte-parole dans sa communauté, mais davantage le remords - vivant ou mort d'un monde voguant sur l'océan des ténèbres ${ }^{43}$ ». Dès lors, comment se ranger sous la bannière d'une nation alors que le seul territoire viable, dans lequel l'écrivain maghrébin puisse résider, se nomme territoire linguistique? Nous habitons donc les lieux par le langage : l'écriture en jeune ruine nous permet d'approcher la ville Tlemcen et de la traduire, au sens de conduire au-delà, faire passer. Les noms restant du Tlemcen des 
années quarante font passer ces "lieux disparus ${ }^{44}$ » que sont le Médresse, Bab Sidi Boumédiène ou la maison du dikhr, et ils nous communiquent une atmosphère. Dib institue donc Tlemcen en tant que lieu des passages, des passes et l'écriture précisément permet cette traversée. Le motif du livre-gigogne peut alors être exhaussé : Tlemcen, l'ouvrage, précisément apparaît comme lieu des passages, entre les temps, nous l'avons $\mathrm{vu}$, et entre les œuvres dont il n'est que l'extension. Par ailleurs, la photographie enserre le texte de Dib de part et d'autre: les deux cahiers circonscrivent la partie textuelle. Le texte fait le passage, fait mouvement d'un bord à l'autre. Dame-Gigogne n'en finit pas de relever ses robes et de convoquer sur la scène de la représentation l'objet littérature ; évoquant un Tlemcen « dont témoignent ces photographies prises en 1946 » et dont il ne resterait peut-être que le nom, Dib apporte un élément de réponse quelques pages plus loin. En effet, il écrit :

Des scènes du Métier à tisser et de Qui se souvient de la mer, se situent dans ce quartier. Sur les mêmes lieux, à l'angle de la place du Médresse et de la rue Bellevue qui, donnant au nord sur toute la plaine mérite bien son nom, il est possible aujourd'hui de s'attabler au café qui a servi de cadre à la nouvelle, Au café ${ }^{45}$.

Suit alors l'incipit en italiques de la nouvelle Au café; la question peut alors être posée : Comment évoquer le Tlemcen des années trente, quarante, cinquante? Qu'est devenue la ville de cette époque, où trouver sa mémoire, où celle-ci s'est-elle réfugiée ? Précisément: dans la lettre, au sein de l'œuvre littéraire. Un renversement s'opère alors : afin de témoigner d'un lieu déterminé qu'est le Médresse, l'écrivain a recours au texte littéraire, lequel a acquis une valeur certaine, s'est trouvé authentifié dans l'histoire de la littérature et de ce fait, authentifie à son tour le lieu par un de ces mots mots de passe - qui ont pouvoir de convocation de l'extrait de l'œuvre littéraire. Et cette traversée des œuvres que pratique l'écriture dibienne constitue à proprement parler une entreprise archéologique quant à la ville nommée Tlemcen. Une quête de l'arkhé interrogeant les temps. En effet, les temporalités sont multiples et aux deux extrêmes temporels que représentent 1946 et 1993, viennent se surajouter les temps de la création. Mobiles. Tour à tour, des extraits de L'Incendie, Au café, Qui se souvient de la mer, Le Désert sans détour ponctuent la méditation dibienne, et donnent à ce portrait de l'écriture une profondeur archéologique singulière. Car en premier lieu, faire le portrait de l'écriture, c'est d'abord faire le portrait du Temps, de ses dépôts. Les caractères romains laissent place aux caractères italiques dans la continuité textuelle, performant à l'œil nu les strates géologiques de l'écriture.

Ils sont là comme, aux mêmes heures d'un après-midi d'août sidéré, il y a longtemps de cela, ils ont assisté à une scène de L'Incendie, le second en date de mes livres. Les deux hommes étaient adossés à un piton de pierre. Le piton, blanc d'un côté, noir de l'autre, gardait la route. Le vieux fellah et le jeune fellah s'étaient réfugiés du côté noir [... $]^{46}$.

Les italiques advenus, c'est comme si le lecteur descendait dans une histoire archéologique de l'écriture visant à abolir - sans l'abolir - la rupture entre le texte citant et le texte cité. En effet, la citation s'insère dans le même paragraphe, fait corps avec le texte actuel, mais avec un corps typographique différent, laissant le dernier mot au texte littéraire cité, couche première de l'écriture. Au fondement. L'évocation de L'Incendie dans cette séquence dite "Les Trois-Frères » déplace le monument naturel évoqué aujourd'hui dans l'Algérie coloniale et lors des velléités d'indépendance qui commencent alors à travailler le pays. L'extrait littéraire du roman L'Incendie convoqué, le lieu-dit voit sa stature rehaussée. Se fait témoin. Il propose au lecteur sa pérennité par le biais de la conjonction comme. Et pose donc un principe d'équivalence temporelle 
à première vue : « Ils sont là comme, aux mêmes heures d'un après-midi sidéré, il y a longtemps de cela, ils ont assisté à une scène de L'Incendie, le second en date de mes livres ${ }^{47}$ " Car il y va du comme défini par Michel Deguy comme d'un principe approximatif ${ }^{48}$. Qui est le nerf de l'inventio poétique. Figure rapprochante/disloquante, les «Trois-Frères" affichent ainsi une identité entamée de différences. Les «TroisFrères » d'aujourd'hui, saisis sur la photographie de Philippe Bordas, ne sont pas tout à fait ceux de L'Incendie, second en date des livres de Dib, au statut de fiction. Ce que le changement de corps typographique performe d'une certaine manière - à l'œil nu. Le comme imprime ici une tension énergétique à la phrase même qui la dissocie d'ellemême et annonce le changement suivant du corps typographique. C'est à un comme oxymorique que le lecteur est confronté : une logique de l'écart préservé se fait jour au cœur du rapprochement opéré. Un parti pris de non-maîtrise, de désappropriation. En ce sens, l'œuvre littéraire nous enjoint aussi à aller y voir : la ville est précisément pour Dib un sujet de réflexion depuis toujours. L'évocation de la guerre d'Algérie était, dans Qui se souvient de la mer, l'occasion d'une investigation de la ville, un instrument de questionnement. L'œuvre littéraire nous donne les moyens de comprendre qu'il y va d'une essence de la ville, d'une manière d'être que précisément le texte littéraire en 1962 ne parvenait pas à cerner. Il avouait déjà l'inanité d'une volonté déterminée de préhension.

II me faut étudier de près les structures de la ville du sous-sol, sans quoi je ne pourrais pas m'adapter, comme il serait souhaitable, aux nouvelles conditions de vie où me voici placé. Que les fondements même de ce second état de l'existence y soient inscrits, j'en suis tout à fait persuadé à présent. À première vue, ces structures ne sont que la réplique de celles de la ville d'en-haut, leur image renversée en quelque sorte et cachée dans les stratifications inférieures ${ }^{49}$.

31 En 1993, l'écrivain Mohammed Dib conclut à l'inanité de cette étude après en avoir reformulé l'hypothèse. L'entreprise topographique est donc une entreprise sans cesse différée, manquée, une entreprise sous voile. Elle reconduit par là même le processus archéologique qui fait d'un lieu un site : à sans cesse re-prendre, finaliser, approfondir afin de l'éclairer autrement, le site en appelle à une écriture précisément se situant dans l'angle, dans le coin. Une écriture qui frôle.

CE PAR QUOI J'AURAIS DÛ CONCLURE - OU COMMENCER ? - [...] c'eût été de dévoiler le visage de cette ville dont à chaque coin de phrase il est question ; c'eût été de le faire apparaître, comparaître, lui, et ce qu'il y a derrière lui $[. . .]^{50}$.

Entreprise sans fin où le visage de cette ville est appelé à com/paraître, toujours avec, aux voiles multiples, l'un dissimulant l'autre, chaque coin cachant ses coins. La phrase fait l'angle : c'est-à-dire se renverse en son contraire, travaille la jonction entre ses deux arêtes. "CE PAR QUOI J'AURAIS DÛ CONCLURE - OU COMMENCER. ${ }^{51}$ " L'ajout syntagmatique entre tirets agrémenté d'un point d'interrogation fait basculer la phrase et inaugure par l'affirmation hypothétique d'un contraire une équivalence, une correspondance absolue; une phrase qui fait l'angle abolit la non-coïncidence des prétendus extrêmes, performe la versatilité du langage et de l'écriture, leur labilité. Ainsi une structure spéculaire est exhaussée : conclure équivaut à commencer, la villed'en-bas dans Qui se souvient de la mer n'est autre que l'image renversée de la ville-d'enhaut. À première vue. En effet, il convient d'être attentif à chaque syntagme de la phrase dibienne. La même difficulté dans les deux ouvrages à circonscrire Tlemcen comporte une différence de taille : le roman se présente comme une transposition fantastique de 
la guerre de libération nationale et n'utilise donc pas le langage usuel. Dib s'en est expliqué dans la postface à Qui se souvient de la mer :

La brusque conscience que j'avais prise à ce moment-là du caractère illimité de l'horreur et, en même temps, de son usure extrêmement rapide est, sans doute aucun, à l'origine de cette écriture de pressentiment et de vision ${ }^{52}$.

La ville évoquée dans Qui se souvient de la mer est donc une représentation hyperbolique, mythique et de plus anonyme : en effet, celle-ci n'est jamais désignée nommément dans le roman même si certains référents laissent à penser qu'il s'agit bien de Tlemcen.

La disposition de la ville : un enchevêtrement de boyaux creusés dans le basalte sur plusieurs étages, avec quelques fentes seulement sur l'air libre mais qu'il est fort difficile de repérer, cette disposition même facilite la communication. [...] Chacun, chaque galerie s'entend, sa vie propre, reliée toutefois par d'invisibles fils au reste, notamment au centre nerveux que nous nommons Médresse, qui ne se limite d'ailleurs pas au seul Médresse mais s'étend à toutes les circonvolutions voisines où un monde en réduction s'abrite ${ }^{53}$.

Ainsi le référent «Médresse » renvoie dans l'esprit de Dib à une cité spécifique, en l'occurrence Tlemcen, mais la cité du roman apparaît comme cité troglodyte, caverne animale, et échappe en somme au règne humain; elle se déshumanise progressivement. Par ailleurs, il convient de noter plus largement que «médresse » est un terme hérité de l'urbanisme arabe et peut donc, à ce titre, se rapporter à quelque autre cité algérienne. Ensuite, la présence de la mer aux portes de la cité dément la référence à Tlemcen et induit le lecteur à y percevoir plutôt Alger. Ce qui se joue ici déborde la dénotation politique et fait de ce roman une quête initiatique spirituelle non moins qu'une initiation politique. La ville Tlemcen sert donc de prétexte à la description de cette ville mythologisée de Qui se souvient de la mer, c'est-à-dire source de fabula, de récits fabuleux. Elle hante la cité littéraire comme une arkhé oubliée. Dans Tlemcen ou les lieux de l'écriture, le référent Tlemcen n'est donc pas davantage traité, évoqué en l'exhaustivité de ses aspects. Ici encore, la ville réelle sert de prétexte au portrait d'une mémoire et d'une écriture. Ce sont deux processus différents de mythologisation mais qui nous montrent que la ville n'est jamais cartographiable, sujette à description potentielle, précisément référençable. Quoi qu'il en soit, le tracé topographique s'apparente ici à l'entreprise littéraire que Dib évoque par ailleurs dans Tlemcen. Une même quête exigeante, éprise d'absolu. Itérable. "Tenter à nouveau l'aventure. Vous ne pouvez dès lors échapper à l'appel de l'œuvre à refaire. Qui sera cette fois parfaite. ${ }^{54}$ » Itérable car l'interrogation garde toujours sa pertinence en 1993 tout comme l'écrivain soulignait déjà en 1962 la possibilité de l'entreprise topographique, possibilité sous menace perpétuelle, portant sa propre suspension puisqu'il s'agit quasiment des dernières lignes du roman: "La tâche surhumaine que représentera le nécessité d'entreprendre l'exploration et le relevé de la cité du sous-sol! Quoi qu'il paraisse, je ne la crois pourtant pas impossible. ${ }^{55}$ " Ainsi, l'œuvre littéraire convoquée au lieu de l'ouvrage est mot de passe, inscription dans la chair même du texte de l'appel impératif en quoi elle mue. Tenter à nouveau l'aventure. La phrase infinitive résonne comme un appel de l'œuvre même, sa voix résonne telle une mise en abyme spéculaire. En ce qui concerne le Médresse évoqué par Dib, une expression est ainsi rappelée par un extrait de la nouvelle «Au café »: le lieu est alors présentifié dans son essence par le texte littéraire. Et rendu à ses temps propres.

Je me suis remémoré le début de cette nouvelle surtout pour retrouver une expression : la place du beylick. Ainsi en effet les Européens appelaient ce qui pour 
nous était le Médresse, en raison de quoi le mot beylick resta cependant longtemps dans mon esprit gros de mépris ${ }^{56}$. une expression figée de la mémoire urbaine. Un spectre, une revenance du lieu. Mais aussi un état socio-politique. En effet, les expressions "médresse » et "place du beylick » rendent compte d'une guerre des langues, des noms, d'une déchirure an sein même du langage, du reflet d'un conflit, d'un différend entre deux communautés ethniques. La langue est connotée et commande des choix qui sont autant de partis pris idéologiques. Et les mots «beylick» et «médresse» fonctionnent comme autant de clefs pour comprendre une période déterminée, une mémoire prise dans des spécificités socio-historiques. L'extrait littéraire convoque une mémoire textuelle mais permet aussi de convoquer un arrière-fond socio-historique. Un processus de mise en abyme s'opère: l'œuvre littéraire est activée, par le biais du titre ou de ses déplacements, dans le texte porteur, le texte $(\mathrm{au})$ présent.

Je me souviens. Il y a longtemps de cela, je me disais aussi : Il me faut étudier de près les structures de la ville du sous-sol, sans quoi je ne pourrais m'adapter, comme il serait souhaitable, aux nouvelles conditions de vie où me voici placé. [...] («Qui se souvient de la mer $»)^{57}$.

Un texte se souvient de l'autre. Ici advient la question de la citation proprement dite en ce qu'elle « donne rendez-vous, elle invite à la lecture, elle sollicite, elle provoque, elle aguiche comme un clin d'œil [...] $]^{58}$ » La citation comme œeil : Dib use de ses ressources afin de faire un clin d'œil au lecteur et met ainsi à contribution sa dimension ludique. Il redécouvre la racine étymologique commune que partagent le jeu et la langue (selon le Robert historique, à l'entrée « jeu » : 1915). Ce que Dib met ainsi en place se nommerait hospitalité, invitation. En effet, un phénomène de porosité gagne les différentes pratiques : au cours de l'ouvrage, le mouvement d'une photographie à l'autre devient celui d'un livre à l'autre, d'un texte à l'autre. Les passerelles ainsi dégagées entre les temps, les textes exhaussent les fragments. C'est en revenir à l'écriture ruiniforme : il n'est que du fragment pour saisir une essence, un parfum. À l'image de la mémoire, fragmentée, fragmentaire. Dib évoque dans Tlemcen le désert comme l'un des éléments fondateurs de la grille de lecture des Algériens, et cite en toute fin un fragment du Désert sans détour, illustrant cette écriture ruiniforme en quête des signes du désert.

Siklist (qui) fait alors :

- Monsieur Hagg-Bar, maintenant je sais pourquoi vous avez pris votre

parapluie avec vous.

Le fort, le gros bonhomme tend l'oreille vers lui :

- Voyons cela. Je t'écoute.

- Pour écrire dans le sable.

- Astucieux. C'est presque ça.

- Presque. Mais pas ça.

- Non.

- Alors, pourquoi? 
- Ah, mon ami, pourquoi!

(Le Désert sans détour ${ }^{59}$ )

38 L'écriture tâtonne, et dévoile progressivement ses axes de lecture; il y va précisément d'une esthétique en face du désert, d'une cosmogonie, d'une quête des signes. "Poésie qui se confondait à son tour avec la recherche du signe, et moins du signe que de la trace du signe ${ }^{60}$. " Précisément, ces signes se nommeraient atlals, signes d'une écriture mystérieuse, indices, augures - ce que rendent particulièrement bien les échanges vifs et lapidaires entre Hagg-Bar et Siklist, lesquels nous mettent sur la piste tout comme les atlals. " Presque. Mais pas ça. " L'adverbe presque performe à la lettre la signification de l'atlal en tant que trace du signe : c'est alors que le processus de mise en abyme se met en place. L'extrait du Désert sans détour ici convoqué apparaît comme un atlal, une grille de lecture, tout comme les autres extraits au long de l'ouvrage remplissent précisément ce rôle, traduisant « une certaine manière d'être de la ville ». Ils jouent ainsi la fonction de source première, de source de la source, et proposent un nouvel état des lieux, un équilibre inversé, donnant à comprendre Tlemcen, ville réelle et ville mythique, depuis l'écriture même. Ces extraits sont annoncés par un mot-symptôme, un mot de passe : la descente dans les strates géologiques de l'écriture s'amorce avant coup. Dans la séquence des «Trois-Frères », le titre du second roman de Dib, L'Incendie, fait office de déclencheur: l'extrait peut alors advenir. Dans une autre séquence intitulée "La maison du dhikr », le terme de metabkka inscrit en italiques convoque quelques lignes plus loin un extrait du roman Qui se souvient de la mer, lequel réinscrit alors ce terme. "Il se décida à descendre les deux marches. Il fit un peu moins sombre dans la metabkha. ${ }^{61} »$ Les extraits-atlals et leurs mots-clefs participent de la mythification de l'entreprise littéraire: de l'œuvre désormais classique. D'un renversement : les Trois-Frères ont assisté à une scène de L'Incendie; le lieu est témoin de l'œuvre littéraire bien plus que l'œuvre ne témoigne du lieu. Faire le portrait d'un lieu, c'est décidément faire le portrait d'une écriture. L'écrivain, faisant état des dépôts du temps et des confrontations des temps entre eux, se fait témoin littéraire. Le témoin littéraire serait celui qui, par la ductilité d'écritures qui composent, nous fait le portrait d'une écriture, et donc de lieux, de temps. Il déhiérarchise les motifs habituels, pose l'écriture comme ce qui est autre-invariablement. C'est-à-dire: s'estrange continûment, joue le supplément de l'image photographique, mais en gagne les coins. Le témoin littéraire se révélerait opérateur d'inversions, jouant de l'ambivalence poétique de la langue afin de déjouer l'identification, l'univocité des définitions qui cèlent. Scellent.

La démarche dibienne est œuvre de mise en relation, mise en œuvre d'une bioéthique qui ne se dépare pas de sa mise en figuration. Un témoin littéraire comme Mohammed Dib signe avec Tlemcen ou les lieux de l'écriture un pseudo-témoignage, c'est-à-dire une "fiction poétique testimoniale»-d'un seul tenant. Faire tenir l'intenable : Tlemcen ou les lieux de l'écriture conteste ses bien-fondés, inscrit continûment en sous-main dans les figures de sa syntaxe l'équivocité de sa quête, l'envers et l'endroit d'une activité nommée écriture, laquelle requiert la plus grande passivité, c'est-à-dire capacité d'accueil. Le témoin littéraire se révèle finalement traducteur, mieux : transducteur, au sens d'un passeur de langues gardant la marque en l'écriture de ces conductions linguistiques. Il opère des connexions, des débordements entre les arts, créant ainsi un troisième corps, au sens où Mireille Calle-Gruber l'entend dans son ouvrage consacré à Hélène Cixous ${ }^{62}$. La scène du livre advient et donne primauté au troisième corps-le poétique. Par un renversement phénoménal, l'écriture gagne son poids de réel et les 
lieux convoqués par l'écrivain se secondarisent, garants d'une réalité première, fondatrice, celle de la littérature. Il ne s'agit certes pas dans Tlemcen ou les lieux de l'écriture d'écrire le Livre des livres mais de composer le portrait d'une écriture en sa mouvance. De donner des clefs, mots de passe, schibboleths d'une écriture qui cherche à capter ses propres traces, la source première, et à se retourner sur elle-même, sur ses propres fondements. À faire advenir le temps du livre. Qui va - en avant.

\section{NOTES}

1. Mohammed Dib, Tlemcen ou la source des sources, Paris, Revue Noire, 1994, p. 109.

2. «Le patio », dans Tlemcen ou la source des sources, ibid., p. 47.

3. Le professeur Thami Benkirane, lors d'un colloque d'hommage à Mohammed Dib («La traversée vers l'autre ») s'étant tenu à Marrakech à l'Institut français et à la Chambre de commerce les 20 et 21 mai 2017, a prononcé une communication, «La photographie de Mohammed Dib», qui a montré combien la pratique photographique de Dib résultait de choix artistiques délibérés. La frontière entre pratique amateuriste et pratique professionnelle tend donc à s'effacer.

4. «L'enfance de l'art », dans Tlemcen ou les lieux de l'écriture, op. cit., p. 80.

5. « Les lieux disparus ", dans ibid., p. 83.

6. Jacques Derrida, Spectres de Marx, Paris, Galilée, 1993, p. 222-256.

7. «Les lieux de l'écriture », dans op. cit., p. 43.

8. Jacques Derrida, « Le ruban de la machine à écrire ", dans Papier machine, Paris, Galilée, 2001, et plus précisément, « Le "seul monument sûr". D’une matérialité sans matière”, p. 105-147.

9. L'Arbre à dires, op. cit., p. 108-109.

10. Walter Benjamin, «Petite histoire de la photographie », dans Euvres II, traduit de l'allemand par Maurice de Gandillac, Pierre Rusch et Rainer Roshlitz, Gallimard, coll « Folio-essais » 2000, p. 308.

11. Tlemcen ou les lieux de l'écriture, op. cit., p. 57.

12. Ibid., p. 58.

13. Ibid.

14. Mireille Calle-Gruber, "L'inlassable réancrage du vécu », entretien avec Claude Simon, dans Le Grand Temps. Essai sur l'œuvre de Claude Simon, Lille, Presses universitaires du Septentrion, coll. «Perspectives », 2004, p. 244-245.

15. Tlemcen ou les lieux de l'écriture, op. cit., p. 58.

16. Ibid.

17. Ibid., p. 87.

18. Ibid., p. 53.

19. Ibid. C'est moi qui souligne.

20. Tlemcen ou les lieux de l'écriture, op. cit., p. 53.

21. «Le patio », dans Tlemcen ou les lieux de l'écriture, op. cit., p. 49.

22. "Abou Madyan », dans ibid., p. 117. C'est moi qui souligne en gras; les italiques sont dans le texte.

23. «Le patio ", dans ibid., p. 47.

24. Ibid., p. 47-48. C'est moi qui souligne en italiques. 
25. Assia Djebar, Le Blanc de l'Algérie, Paris, Albin Michel, 1996, p. 276.

26. « Des enfants plein la ville », dans Tlemcen, op. cit., p. 65.

27. Ibidem.

28. Ibidem.

29. Roland Barthes, La Chambre claire, Paris, Cahiers du cinéma/Gallimard/Le Seuil, 1980, p. 30. C'est moi qui souligne en italiques.

30. L'Arbre à dires, op. cit., p. 107.

31. Paul Ricœur, La Métaphore vive [1975], Paris, « Points-Essais », 1997, p. 64. L'italique et les guillemets sont dans le texte.

32. «Le patio ", dans Tlemcen, op. cit., p. 51.

33. L'Arbre à dires, op. cit., p. 111.

34. Ibid.

35. Ibid., p. 49.

36. « La ville », dans ibid., p. 125.

37. Mohammed Dib, Qui se souvient de la mer, " postface », Paris, Le Seuil, 1962, p. 191. Les italiques sont dans le texte; c'est moi qui souligne en gras.

38. Ibid. Les italiques sont dans le texte; c'est moi qui souligne en gras.

39. Pierre Reverdy, Le Gant de crin, Paris, Plon, 1927, cité par André Breton dans Le Manifeste du surréalisme, Gallimard, coll. « La Pléiade », tome I, 1988, p. 324.

40. «Le Médresse », dans Tlemcen, op. cit., p. 83.

41. Assia Djebar, Le Blanc de l'Algérie, op. cit., p. 276.

42. Assia Djebar, «L'écriture de l'expatriation », dans Ces voix qui m'assiègent, Paris, Albin Michel, 1999, p. 213.

43. Ibid., p. 216.

44. « Le Médresse », dans Tlemcen, op. cit., p. 83.

45. « Les lieux disparus », dans ibid., p. 85-86.

46. «Les Trois-Frères ", dans ibid., p. 58. Les italiques sont dans le texte.

47. Ibid. Je souligne en gras pour différencier d'avec les italiques que Dib utilise pour citer les extraits de ses œuvres.

48. Michel Deguy, La poésie n'est pas seule. Court traité de poétique, Paris, Le Seuil, coll. « Fiction \& Cie », 1987, notamment « Feuillure $3:$ Il y a comme et comme », p. 88-93.

49. Qui se souvient de la mer, op. cit., p. 185, cité dans Tlemcen, op. cit., p. 125. Les italiques sont dans le texte.

50. Ibid. C'est moi qui souligne en gras. Les capitales sont dans le texte.

51. Ibid. Les italiques sont dans le texte.

52. "Postface ", Qui se souvient de la mer, op. cit., p. 189.

53. Ibid., p. 24. C'est moi qui souligne en italiques.

54. « Les impasses de l'écriture », dans Tlemcen, op. cit., p. 99.

55. Qui se souvient de la mer, op. cit., p. 187.

56. « Le médresse », dans « Les lieux disparus ", dans Tlemcen, op. cit., p. 86.

57. « La ville », dans ibid., p. 125. Les italiques sont dans le texte. C'est moi qui souligne en gras.

58. Antoine Compagnon, La Seconde Main, ou le travail de la citation, Paris, Le Seuil, 1979, p. 23.

59. Mohammed Dib, Le Désert dans détour [1992], cité dans Tlemcen, op. cit., p. 108. C'est moi qui souligne en italiques.

60. Ibid.

61. Qui se souvient de la mer, op. cit., dans Tlemcen, op. cit., p. 90. Les italiques sont dans le texte. C'est moi qui souligne en gras.

62. Mireille Calle-Gruber, Du café à l'éternité. Hélène Cixous à l'œuvre, Paris, Galilée, 2002. 


\section{RÉSUMÉS}

L'ouvrage Tlemcen ou les lieux de l'écriture de l'écrivain algérien d'expression française Mohammed Dib, publié en 1993, est une œuvre singulière : associant photographies prises par le jeune Dib au lendemain de la guerre aux photographies prises quasiment cinquante ans plus tard par le photographie français Philippe Bordas, l'opus est prétexte pour l'écrivain à une exploration par l'écriture d'une ville spécifique, Tlemcen, et des strates mémorielles qui la composent. Mais c'est aussi à une exploration de l'œuvre dibienne que cet ouvrage invite : faire le portrait de Tlemcen, entreprise par essence infinie, consiste à faire le portrait d'une écriture.

The work of the Algerian writer of French expression Mohammed Dib, entitled Tlemcen ou les lieux de l'écriture and published in 1993, is a singular opus : associating pictures taken by the young Dib just after the Second World War to pictures taken almost fifty years later by French photographer Philippe Bordas, the book is a pretext for the writer in an exploration by the writing of a specific city, Tlemcen, and strata memory which make it up. But it is also in an exploration of the dibian work that this opus invites : to paint the portrait of Tlemcen, undertaken in essence infinite, consists in painting the portrait of a writing.

\section{INDEX}

Mots-clés : Tlemcen, Photographie, Mémoire, Ville, Écriture, Portrait

\section{AUTEUR}

\section{HERVÉ SANSON}

Hervé Sanson, docteur ès lettres modernes, spécialiste des littératures francophones du Maghreb, membre associé à l'ITEM, a collaboré aux Portraits d'Albert Memmi (CNRS éditions, 2015) sous la direction du professeur Guy Dugas. Il est l'éditeur scientifique du recueil d'essais d'Albert Memmi, Penser à vif, de la colonisation à la laïcité, paru en 2017 chez Non Lieu. Il prépare actuellement une monographie sur l'oeuvre de Dib pour les éditions Honoré Champion. 